J Ästhet Chir 2019 - 12:135-141 https://doi.org/10.1007/s12631-019-0186-3 Online publiziert: 1. April 2019

(c) Der/die Autor(en) 2019

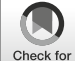

Frank G. Neidel

Spezialpraxis Haartransplantation HAIRDOC, Düsseldorf, Deutschland

\title{
Haartransplantation: Welche Technik für welche Patienten?
}

\section{Zeitliche Einordnung der Operationstechniken}

Die Haartransplantation ist als Umverteilung Dihydrotestosteron(DHE)resistenter Haarfollikel aus dem so genannten Haarkranz in kahle Stellen, zum Beispiel bei androgenetischer Alopezie (AGA) oder bei narbigen Alopezien verschiedener Genese seit über 70 Jahren als wirksame und dauerhafte Therapie bekannt [2, 7, 12-14].

Anfänglich „punchte“ man Hautinseln mit Durchmesser von 3-5 mm aus der Spenderzone (Haarkranz) heraus. Die Entnahmestellen blieben offen oder wurden vernäht und die Makrotransplantate (Grafts) in Originalgröße in die Kahlstellen umverteilt [3, 16]. Alopezische Areale waren damit zwar wieder lückenhaft behaart, unweigerlich wurde jedoch ein deutlicher Büscheleffekt sichtbar. Hohen ästhetischen Ansprüchen konnte das Resultat nicht genügen (- Abb. 1 und 2).

In den 1980iger-Jahren wurden Standardtransplantate (Hautinseln mit 3-5 mm Durchmesser) geviertelt oder gesechstelt, also präparatorisch verkleinert, so dass die Büschel nicht mehr so deutlich sichtbar waren. Es begann die Ära der Minigrafts. Minigrafts wurden für die Gestaltung des Haaransatzes sogar in geringer Zahl zu Einzelhaartransplantaten (Mikrografts) präpariert $[5,6]$.

Beide Methoden, Standardgrafttransplantation und Mini-/Mikrografttransplantation begrenzten sich durch relativ hohe Verluste an Haarwurzeln und größere Vernarbungen im Spendergebiet.

Mit Einführung von Mikroskopen in den frühen 1990iger-Jahren zur Präparation von Haarwurzeln wurde zum einen die Dissektions-, also Verlustrate an Haarwurzeln gesenkt. Zum anderen entnahm man am Hinterkopf nicht mehr nur Hautinseln, sondern einen mehr oder weniger langen und breiten Hautstreifen, der sehr viele Haarwurzeln enthielt. Man war in der Lage, daraus große Mengen an Haartransplantaten relativ verlustarm $\mathrm{zu}$ vereinzeln $[8,9,16]$. Es wurde versucht, immer höhere Transplantatzahlen $\mathrm{zu}$ verpflanzen und damit eine dichtere Bedeckung der Kopfhaut zu erreichen bzw. die Behandlungsfläche zu vergrößern. Das Management der Narben am Hinterkopf wurde dabei weitestgehend außer Acht gelassen, auch, weil man die verbliebenen Resthaare ganz gut zum Überkämmen breiter Narben nutzen konnte. Die Patienten tolerierten die entstandenen Narben am Haarkranz weitestgehend (- Abb. 3 und 4).

Mit Beginn des 21. Jahrhunderts wurden Kurzhaarschnitte modern und somit wurden die Entnahmenarben auch mehr sichtbar. Über diverse Kommunikationskanäle im Internet wurden solche Narben dargestellt, und es entwickelte sich ein Bewusstsein für möglichst unsichtbare Narben im Haarkranzbereich. Spezielle Nahttechniken zum Wundverschluss wurden entwickelt („trichophytic closure" nach Marzola oder Frechet) [16]. Somit konnte unter bestimmten Voraussetzungen mit der richtigen Entnahme- und Nahttechnik eine auch bei Kurzhaarschnitt fast unsichtbare lineare Narbe erreicht werden bei akzeptabler Transplantatzahl von 2000 bis 3000. Nicht immer konnte man von optimaler Wundheilung und fachgerechtem Wundverschluss ausgehen, weshalb durch ungünstige Narbenbildung, besonders nach Mehrfachoperationen, die

Transplantation $=$ FUT 


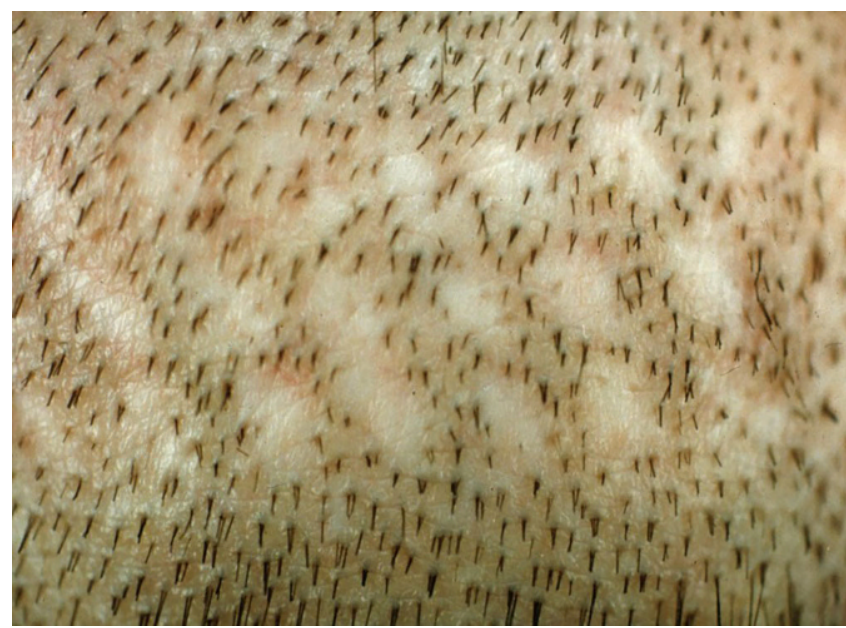

Abb. 1 A Hautinseln mit Durchmesser von 3-5 mm wurden aus dem Haarkranz gebohrt. Man nennt sie Standardtransplantate nach Perlenkettenentnahme, weil der Narbenverlauf wie eine Perlenkette anmutet. (@ F. Neidel)

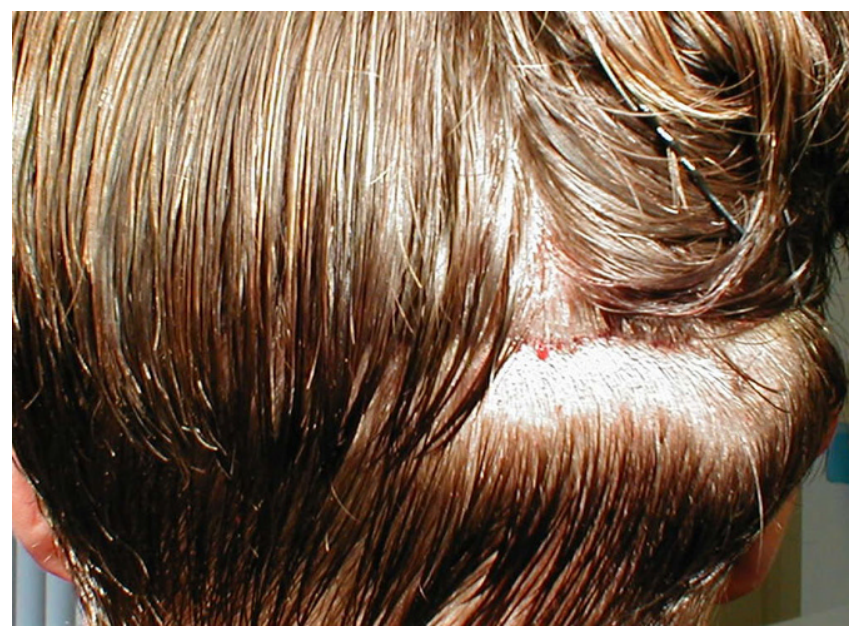

Abb. 3 A Gerade vernähte Entnahmestelle nach Streifenentnahme. Die Resthaare bleiben lang und können die Entnahmestelle überdecken. (৫ F. Neidel)

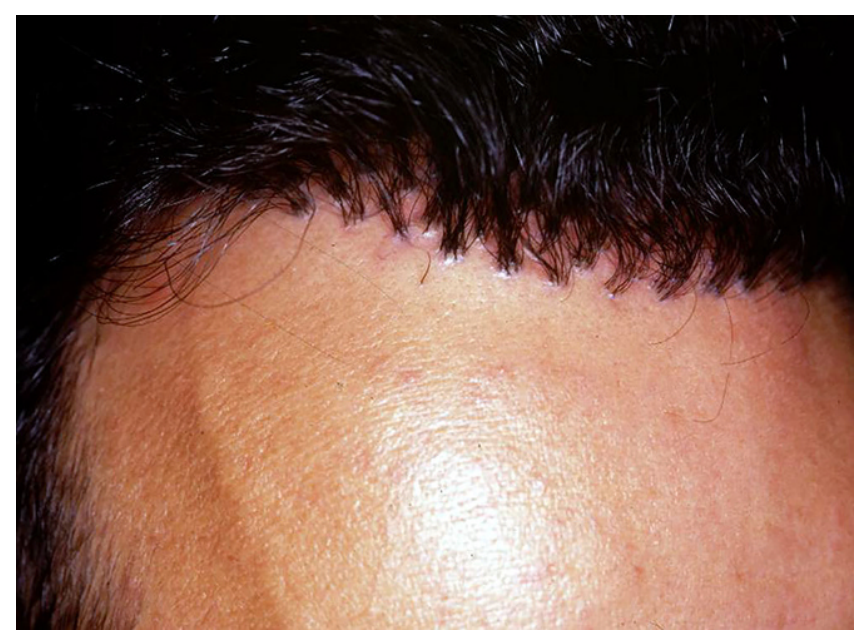

Abb. 2 A "Puppenkopfeffekt" = unnatürliches Aussehen durch Transplantation von Hautinseln (Makrografts) mit Durchmesser 3-5 mm aus dem hinteren Haarkranz. (๑ F. Neidel)

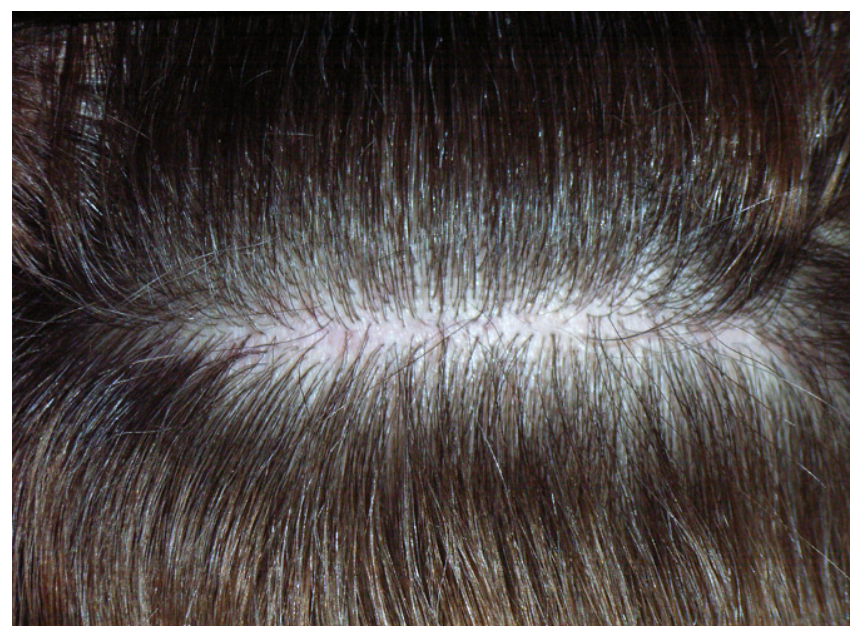

Abb. 4 \& Nach optimaler Wundheilung ist die Narbe bei langem Haar kaum oder gar nicht sichtbar. (৫ F. Neidel)
Streifenentnahme in Misskredit geriet (- Abb. 5 und 6).

Durch mikroskopische Präparation und damit zwangsläufig auch genauere optische Analyse der Spenderfläche erkannte man, dass Haarwurzeln in kleinen Gruppen aus der Haut herauswachsen und als anatomische Einheit zu betrachten sind. Diese Erkenntnis wurde histologisch bestätigt. Man nannte diese Haareinheiten „Follicular Units“. Der Begriff der Follicular-Unit-Transplantation (FUT) entstand ([1]; • Abb. 7).

Im Jahr 2002 nutze Dr. Bill Rassmann die Weiterentwicklung der Mikrochirurgie und benutzte sehr kleine Hohlbohrer von 1-1,5 mm Durchmesser anstatt der
Standardbohrer von 3-5 mm. Dadurch konnte er „Follicular Units“ direkt entnehmen. Er nannte das Verfahren der Einzelentnahme „FOX-procedure“. Mit Bekanntwerden und weiterer Verbreitung dieser Entnahmetechnik entstand der Begriff der FUE-Entnahmetechnik („Follicular-Unit-Extraktion“) [15]. Der Begriff wurde 2018 konkretisiert und ersetzt durch den Begriff der „FollicularUnit-Exzision“. Damit wird klar definiert, dass es sich um ein Herausschneiden von Haarwurzeln, also um ein invasives Vorgehen handelt, was nach unserer Rechtsauffassung Ärzten vorbehalten ist [11].
Merke. Die seit 2002 bekannte und in den Folgejahren stets weiterentwickelte Einzelentnahme der „Follicular Units“ aus dem hinteren und seitlichen Haarkranz wird per definitionem als „Follicular-Unit-Exzision“ bezeichnet. Nach unserer Rechtsauffassung ist dieser invasive Vorgang approbierten Ärzten vorbehalten bzw. kann unter Aufsicht approbierter Ärzte roboterassistiert durchgeführt werden [10].

\section{Aktuelle Situation}

Die FUE-Entnahmetechnik entwickelte sich im letzten Jahrzehnt rasant weiter sowohl in puncto Instrumentation als 
auch in puncto „Lernkurve“ der Operateure. Verpflanzte man anfänglich bis zu 1000 „Follicular Units“ pro Sitzung an einem Tag, so ist heute unter optimalen Bedingungen und hoher Haardichte beim Patienten eine Zahl bis zu 2500 und mehr möglich. Auch Haarroboter helfen bei der Entnahme der "Follicular Units" und schützen den Operateur vor Ermüdung.

Trotzdem soll an dieser Stelle davor gewarnt werden, Transplantatzahlen ins „Unermessliche“ zu steigern. Denn: Die Spenderhaarfläche ist begrenzt, die Spenderhaardichte ist individuell verschieden. Nur nach genauer Analyse der Größe von Spenderfläche und Haarfollikelgruppendichte kann man Aussagen über die Gesamtmenge transplantierbarer Haarwurzeln machen.

Auch bei der Einzelentnahme können sichtbare Narben resultieren, z. B. bei inadäquatem Instrumentarium und beim „lernenden Operateur". Bei zu dichter Entnahme und zu hoher Transplantatzahl kann der gesamte Hinterkopf ver- narben, es wachsen kaum noch Haare, und der Patient gilt als "abgeerntet" mit sichtbarer Narbenbildung (• Abb. 8). Bei nachhaltiger Entnahmetechnik heilen die Wunden sehr schnell ab, die Narbenbildung ist diskret und später gar nicht oder kaum sichtbar (• Abb. 9).

Bei Streifenentnahme- als auch bei FUE-Entnahmetechnik sind die Resultate gleichwertig und genügen höchsten ästhetischen Ansprüchen sowohl makround mikroskopisch (• Abb. 10a, b). Der natürliche Wunsch eines jeden Patienten ist: So viel wie möglich Haarwurzeln transplantieren, so schonend wie möglich transplantieren, so schmerzarm wie möglich transplantieren, so nebenwirkungsarm wie möglich arbeiten, damit die Wiederherstellungsphase möglichst kurz ist.

Merke. Gerade wegen kommerzieller Versprechen im Internet, die rein medizinisch betrachtet oft falsch sind, haben wir Ärzte eine Aufklärungspflicht gegenüber unseren Patienten, der wir in vollem
Umfang nachkommen sollen. $\mathrm{Zu}$ einem guten ärztlichen Beratungsgespräch gehören die Analyse der Spenderhaarquantität und -qualität, ein zukunftsweisendes Behandlungskonzept und die richtige Wahl des anzuwendenden Operationsund Entnahmeverfahrens.

Die Entnahmetechniken verteilen sich derzeit weltweit mit rund $44 \%$ auf Streifenentnahme mit anschließender mikroskopischer Präparation (FUT) und zu 52\% auf Einzelentnahme (FUE) mit direkter Reimplantation im 1:1-Verfahren, rund $4 \%$ sind kombinierte Verfahren [4]. Beide Entnahmetechniken sind im Prinzip gleichwertig; Voraussetzungen sind fachgerechte Ausführung, trainiertes Team und moderne Instrumentation. Viele subjektiv gefärbte Meinungen "pro oder contra" einer Technik müssen heute revidiert werden. Noch immer kursieren Mythen durch das Internet und stellen die eine oder die andere Entnahmetechnik negativ dar. Das ist angesichts guter Resultate beider Methoden nicht länger gerechtfertigt.

Hier steht eine Anzeige. 
Am Ende führt der „Kampf der Entnahmetechniken“ zu völlig verunsicherten Patienten, die sich dann eher gegen eine Haartransplantation entscheiden, und das kann man durchaus verstehen.

Merke. Mit heutigem Stand der Technik und bei lege artis angewandten Operationsverfahren sind im Resultat sowohl die Streifenentnahmetechnik (FUT) als auch die Einzelentnahmetechnik (FUE) als völlig gleichberechtigt anzusehen. Mit beiden Techniken lassen sich hervorragende Resultate erzielen.

Nach welchen Kriterien soll nun der Arzt entscheiden, welche Entnahmetechnik für den Patienten besser bzw. empfehlenswerter ist?

\section{Kriterien zur Empfehlung für FUE-Entnahmetechnik (Einzelentnahme)}

\section{Männer, jünger als 30 Jahre (+/-)} Begründung: In jungen Jahren trifft Haarausfall die Patienten besonders hart, man reagiert oft panisch und neigt zu übereilten Entscheidungen. Auch ist völlig unklar, welche Frisur man vielleicht später einmal tragen wird, besonders bei starker Progredienz des Haarverlustes. Und oftmals ist für den Patienten über 30, der in einer stabilen Beziehung lebt, vielleicht schon Vater von Kindern ist, das Aussehen eher zweitrangig geworden. Somit tendiert man viel leichter zu einem Kurzhaarschnitt oder gar zur Glatze. Hatte man in jungen Jahren eine Haartransplantation mit Streifenentnahmetechnik, dann wird die strichförmige Narbe vielleicht sichtbar sein, und man wird wiederholt darauf angesprochen. Das führt zu Frustration.

2. Männer mit Kurzhaarschnitt (unter 0,5-1 cm Länge)

Begründung: Das Haar ist zu kurz, um Naht und Narbenrötung direkt nach der Behandlung abzudecken. Später wird die Narbe unter Umständen gar nicht oder nur gering sichtbar sein, doch das sollte nicht als Kriterium dienen.

3. Männer mit sehr dunklem, kurzem Haar (z. B. schwarz) und heller Haut

J Ästhet Chir 2019 - 12:135-141 https://doi.org/10.1007/s12631-019-0186-3

(c) Der/die Autor(en) 2019

\section{F. G. Neidel}

\section{Haartransplantation: Welche Technik für welche Patienten?}

\section{Zusammenfassung}

Während der Transplantationsprozess von Haarwurzeln in ehemals behaarte Kahlstellen weitestgehend standardisiert ist und sich über die Jahre kaum verändert hat, haben sich in den letzten 15 Jahren 2 verschiedene Entnahmetechniken der Haarwurzeln aus dem Haarkranz durchgesetzt: Einmal die renommierte Streifenentnahme mit anschließender mikroskopischer Vereinzelung der "Follicular Units", zum zweiten die seit 2002 erstmals beschriebene Einzelexzision der "Follicular Units", FUE-Technik. Beide Techniken bieten Vor- und Nachteile für den Patienten. Durch Marketingstrategien im Internet sind Patienten oft verunsichert und wissen nicht, für welche Entnahmemethode sie sich entscheiden sollen. Aufgrund empirischer Erfahrungen sollte man bei Patienten bis zum 30. Lebensjahr und bei Patienten mit Kurzhaarschnitt (weniger als $1 \mathrm{~cm}$ Haarlänge) sowie dünnem und durchscheinendem Haar die FUE-Methode empfehlen. Auch bei Ängsten vor "Schneiden, chirurgischer Naht" etc. ist die FUE-Technik die Methode der Wahl. Patienten, die aus beruflichen oder persönlichen Gründen niemals ihre Haare kurz rasieren würden, bietet sich die Strip-Entnahmetechnik mit resorbierbarer Naht an. Das trifft natürlich auch auf alle Patienten zu, die immer ihr Haar lang tragen möchten. Dazu zählen in den meisten Fällen auch weibliche Patienten. Wichtig für den Arzt in der Haarsprechstunde: Wird eine Methode schlechtgeredet oder vorrangig Negatives darüber in den Vordergrund gestellt, verunsichert man den Patienten allgemein. Ärzten, die nur eine Entnahmetechnik beherrschen, wird dringend das Erlernen der Alternativmethode empfohlen. Der Patient wird sich immer dort am sichersten und besten fühlen, wo der Arzt alle derzeit gängigen Operationsverfahren beherrscht. Es gehört zur ärztlichen Kunst und ist eine "conditio sine qua non", die Entnahmetechniken selbst zu beherrschen und anzuwenden und diese nicht an Personal zu delegieren.

\section{Schlüsselwörter}

Haartransplantation · FUE · FUT · Entnahmetechniken · Patientenauswahl

\section{Hair transplantation: which technique for which patient?}

Abstract

While the process of transplanting hair roots into bald areas which were previously piliferous is extensively standardized and has hardly changed over the years, in the last 15 years 2 different harvesting techniques of hair roots have prevailed. Firstly, the renowned strip harvesting with subsequent microscopic isolation of the follicular units and secondly, the individual excision of the follicular units (FUE technique) described for the first time in 2002. Both techniques offer advantages and disadvantages for the patient. Patients are often unsettled by marketing strategies on the Internet and do not know which harvesting technique to choose. Based on empirical experience, the FUE method should be recommended for patients up to 30 years of age and for patients with a short haircut (less than $1 \mathrm{~cm}$ hair length) and thin or translucent hair. The FUE technique is also the method of choice for fear of "cutting and surgical suturing" etc. Patients who would never have a short haircut for professional or personal reasons can use the strip harvesting technique with resorbable sutures. Of course, this also applies to all patients who always want to wear their hair long. In most cases, this also includes female patients. Important for the physician during the hair consultation: if a method has a poor reputation or negative aspects are given priority, the patient is generally made insecure. It is strongly recommended that physicians who only master one harvesting technique should learn the alternative method. The patient will always feel safest and best where the physician has mastered all currently available surgical procedures. It belongs to the medical skills and is a conditio sine qua non to master and apply the harvesting techniques oneself and not to delegate them to medical assistants.

Keywords

Hair transplantation · FUE · FUT · Harvesting techniques · Patient selection 


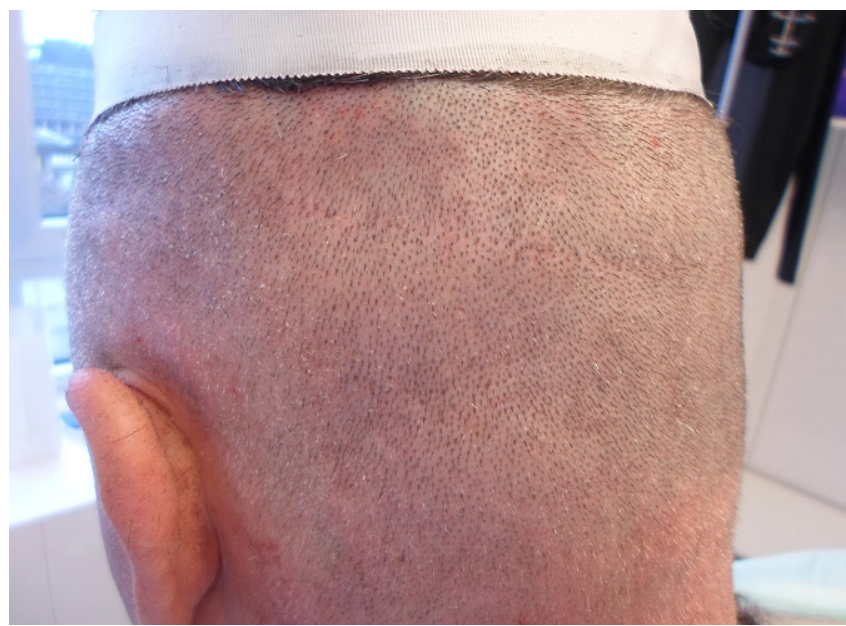

Abb. 5 ॥ Optimal versorgte Narbe nach Streifenentnahme mit Durchwachsungsnaht („trichophytic closure“), keine Dehiszenz erkennbar, linearer Narbenverlauf auch bei Glatze schwer erkennbar. (@ F. Neidel)

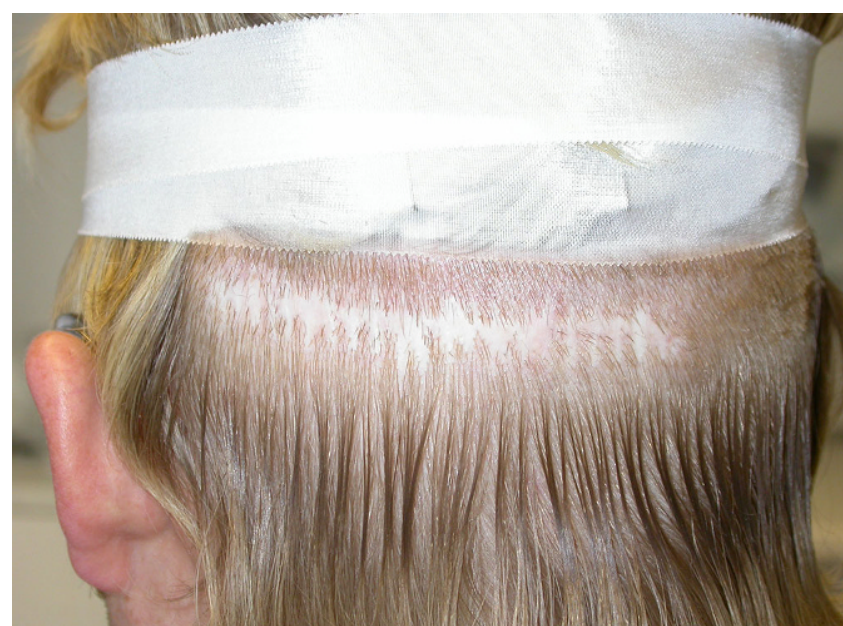

Abb. $6 \Delta$ Schlecht versorgte Narbe nach Streifenentnahme, „Strickleitermuster" wegen durchgreifender Nähte, Narbe wäre bei Glatze deutlich sichtbar. (@ F. Neidel)

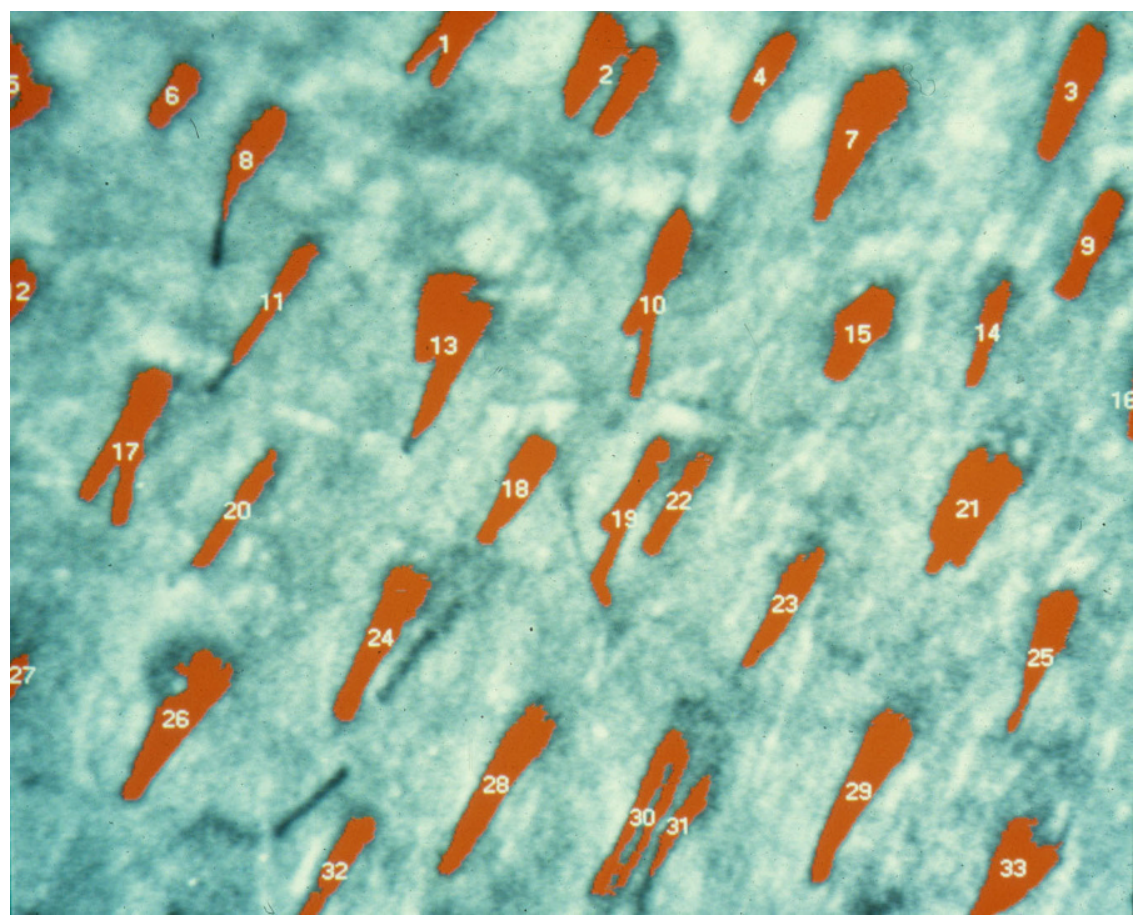

Abb. 7 ॥ „Follicular Units“ können genau pro Flächeneinheit gezählt und/oder computergestützt analysiert werden. (৫ F. Neidel)

Begründung: Je dunkler die Haarfarbe und je heller die Haut, desto eher scheint auch eine helle oder gar weiße strichförmige Narbe durch das kurze Haar hindurch. Cave: auch große punktförmige Entnahmepunkte nach FUE-Behandlung können hier sichtbar werden.

4. Männer und Frauen, die Angst vor „Schneiden, Skalpell, Naht" haben
Begründung: Besonders nach vielen Voroperationen, die alle mit „Schnitten" einhergingen, sind die Patienten ängstlicher und „dünnhäutig“ bezüglich „Schneiden“ geworden. Wer Angst vor einem Schnitt bzw. auch einer Naht hat, dem sollte man diese Angst nicht ausreden wollen.

5. Frauen, die nur wenige Haartransplantate benötigen $(\leq 800+/-)$ und/ oder Kurzhaarschnitt

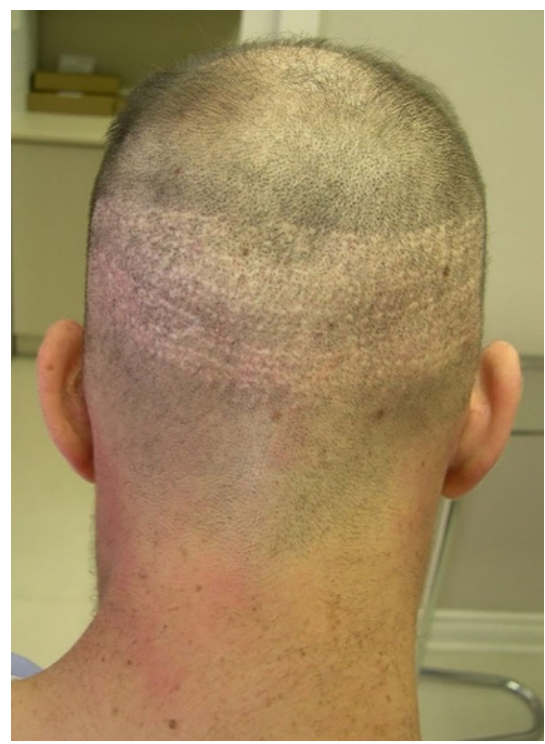

Abb. 8 ॥ Sichtbare Narben nach FUE-Technik. Hier wurde ein zu großer Durchmesser des Entnahmeinstruments gewählt und das Entnahmemuster zu dicht und perlenkettenartig angelegt. FUE Follicular-Unit-Exzision. (৫ F. Neidel)

Begründung: Ein Schnitt mit Naht belastet mehr. Bei sehr kurzem Haar würde die Naht für Tage und Wochen sichtbar sein.

\section{Kriterien zur Empfehlung für Streifenentnahme (FUT)}

1. Patienten über 40 Jahre (+/-), die immer schon langes Haar tragen 


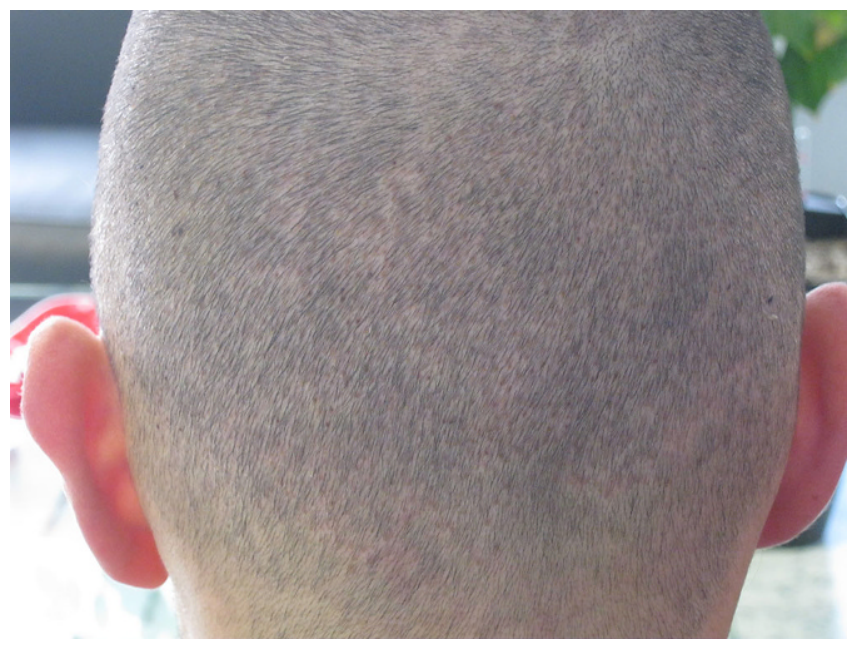

Abb. 9 A Bereits nach 1 Woche sind bei fachgerechter Ausführung der FUETechnik die Entnahmepunkte kaum noch sichtbar, da randomisiert verteilt. FUE Follicular-Unit-Exzision. (@ F. Neidel)

Begründung: Diese Männer würden sich nie für eine Glatze entscheiden und würden dann eher auf eine Haartransplantation verzichten, wenn sie die Haare abrasieren müssten.

2. Patienten, die eine große Menge an Haartransplantaten benötigen Begründung: Hier ist es sinnvoll, zunächst mit der Streifenmethode möglichst viele Haarwurzeln zu gewinnen. Später kann man dann im Nacken- und am oberen Haarkranz ergänzend Einzelentnahmen durchführen. Somit erhöht man die Gesamtzahl transplantierbarer Haarwurzeln, ohne das optische Erscheinungsbild des Haarkranzes wesentlich zu verändern. Alternativ kann auch im Konzept mit FUE begonnen werden und anschließend im Jahresabstand zur Streifenentnahme gewechselt werden.

3. Patienten in prädisponierten gesellschaftlichen Positionen mit viel Publikumsverkehr

Begründung: Das Resthaar kann lang belassen werden. Damit lässt sich die Naht nach Stripentnahme perfekt überkämmen. Auch später sieht man bei normal langem Haar keine Narbe, vor allem aber bleibt die Haardichte annähernd gleich.

4. Frauen, die Transplantatmengen über 800 (+/-) „Follicular Units“ benötigen Begründung: eine großflächige Kahlrasur beeinträchtigt extrem das
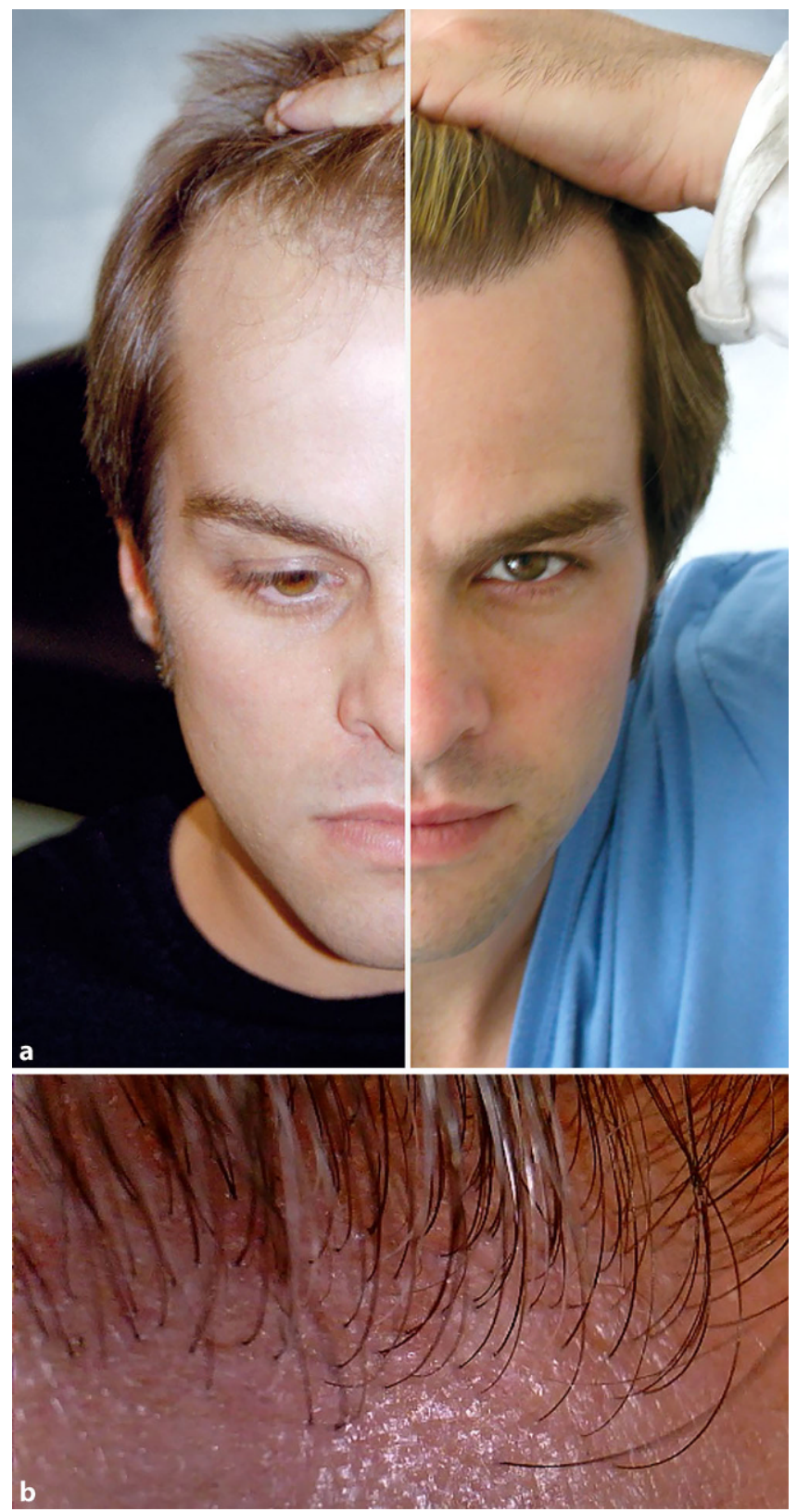

Abb. $10 \Delta$ a Wunsch nach Rekonstruktion des Haaransatzes vor der Haartransplantation, b Resultat des mit „Follicular Units" rekonstruierten Haaransatzes (FUT) im Vergleich u vorher

Wohlbefinden dieser Patientinnen, vergleichbar mit dem Haarausfall nach Chemotherapie.

Merke. Wichtigstes Entscheidungskriterium für die Wahl der Entnahmetechnik ist die damit verbundene Frage an den Patienten: „Können Sie sich vorstellen, 4 bis 12 Wochen Glatze bzw. sehr kurzes Haar zu tragen?“. Wenn „JA“, dann ist die FUE-Technik möglich; wenn „NEIN“, dann wird die Streifenentnahme (FUT) empfohlen.

Hier gibt es auch keine Kompromisse. Ein „wenig kurzes Haar“ erlaubt keine Einzelentnahme. Auch bei kleineren kahl rasierten Flächen lassen sich nur geringe Transplantatmengen entnehmen. Die Haarlänge muss zwingend $1,2-1,4 \mathrm{~mm}$ betragen. Langhaareinzelentnahmen werden zwar bereits durchgeführt, jedoch sind diese sehr 
aufwendig und erbringen noch nicht die gewünschten Transplantatmengen.

\section{Tipp für die Praxis}

Je jünger der Patient und je flexibler/ weicher seine Haut ist (das bedeutet relativ wenig Kollagenfasern), desto stärker ist die Neigung zur Wunddehiszenz mit dauerhafter Breitnarbenbildung nach Streifenentnahme.

Je flexibler und weicher die Haut und je kräftiger die Haare, desto einfacher und besser ist die FUE-Entnahme möglich.

Je fester die Haut und je höher der Kollagenfaseranteil (straff, unnachgiebig), desto schwieriger ist die FUE-Entnahme, besonders bei sehr dünnem und feinem Haar, weil damit die Reibungs- und Scherkräfte eher zu Transplantatbruch und Dissektion führen. Hier ist die Streifenentnahme hervorragend möglich, weil fast immer eine kaum oder wenig sichtbare strichförmige Narbe resultiert. Voraussetzung ist eine maximale Streifenbreite von $1,0-1,3 \mathrm{~cm}$. Optimal ist eine Streifenbreite von $1,0 \mathrm{~cm}$ bei variabler Streifenlänge, je nach Bedarf an Transplantaten.

\section{Fazit}

Dem Arzt obliegt es, am Ende gemeinsam mit dem Patienten die Entnahmetechnik zu entscheiden. Dabei spielen Erfahrung, technische und personelle Ausstattung sowie manuelle Fertigkeiten die entscheidende Rolle. Die derzeit angewandten Techniken sind gut etabliert und dürfen nicht argumentativ in Misskredit gebracht werden, weil das den Patienten eher verunsichert. Vielmehr sollte man seine Möglichkeiten und Grenzen der Therapie kennen und gegebenenfalls schwierige Patienten ablehnen und zum Spezialisten überweisen.

\section{Korrespondenzadresse}

Dr. med. Frank G. Neidel
Spezialpraxis Haartransplan-
tation HAIRDOC
Königsallee 30, 40212 Düssel-
dorf, Deutschland
info@hairdoc.de

\section{Einhaltung ethischer Richtlinien}

Interessenkonflikt. F.G. Neidel gibt an, dass kein Interessenkonflikt besteht.

Für diesen Beitrag wurden vom Autor keine Studien an Menschen oder Tieren durchgeführt. Für die aufgeführten Studien gelten die jeweils dort angegebenen ethischen Richtlinien. Für Bildmaterial oder anderweitige Angaben innerhalb des Manuskripts, über die Patienten zu identifizieren sind, liegt von ihnen und/ oder ihren gesetzlichen Vertretern eine schriftliche Einwilligung vor.

Open Access Dieser Artikel wird unter der Creative Commons Namensnennung 4.0 International Lizenz (http://creativecommons.org/licenses/by/4.0/deed. de) veröffentlicht, welche die Nutzung, Vervielfältigung, Bearbeitung, Verbreitung und Wiedergabe in jeglichem Medium und Format erlaubt, sofern Sie den/die ursprünglichen Autor(en) und die Quelle ordnungsgemäßnennen, einen Linkzur Creative Commons Lizenz beifügen und angeben, ob Änderungen vorgenommen wurden.

\section{Literatur}

1. Bernstein RM, Rassmann WR, Seager D, Shapiro R, Cooley JE, Norwood OT, Stough DB, Beehner M, Arnold J, Limmer BL, Avram MR, McClellan RE, Rose PT, Blugerman G, Gandelman M, Cotterill PC, Haber R, Jones R, Vogel JE, Moy RL, Unger WP (1998) Standardizing the classifikation and description of follicular unit transplantation and mini-micrografting techniques. Dermatolog Surg 24:957-963

2. Hamilton JB (1951) Patterned loss of hair in man; types and incidence. Ann Ny Acad Sci 53(3):708-728

3. Farber GA (1982) The punch scalp graft. Clin Plast Surg 9(2):207-220

4. ISHRS (2017) Pracitice census 2017. http://www. ishrs.org/statistics-research.htm. Zugegriffen: 25 Febr. 2019

5. Lucas MWG (1988) The use of minigrafts in hair transplantation surgery. J Dermatol Surg Oncol 14:1389-1392

6. Marritt E (1984) Single-hair transplantation for hairline refinement: A practical solution. JDermatol Surg Oncol 10:962-963

7. Neidel FG (1995) Eigenhaartransplantation bei narbigen Alopezien. Zentralbl Haut- und Geschlechtskrankheiten. Springer, Bd. 166, S325-326

8. Neidel FG (2003) Operative Techniken bei Haarverlust. In: Dirschka T, Sommer B, Usmiani J (Hrsg) Leitfaden Ästhetische Medizin, 1. Aufl. Urban \& Fischer, München, Jena, S247-284

9. Neidel FG (2015) Eigenhaartransplantation. In: Kardorff B (Hrsg) Selbstzahlerleistungen in der Dermatologie und der ästhetischen Medizin, 2. Aufl. Springer, Berlin, Heidelberg, S447-467

10. Neidel FG (2017) 2 Jahre Erfahrung mit dem Haarroboter ARTAS. Haut 4:194-196

11. Neidel FG, Finner A (2015) GÄCD Leitlinien Haartransplantation unter: http://www.gacd.de/ haartransplantation.php. Abgerufen Am 6(2017):15

12. Norwood OT (1975) Male pattern baldness: classification and incidence. South Med J 68(11):1359-1365
13. Okuda S (1939) The study of clinical experiments of hair transplantation. Jpn J Dermatol Urol 46:135

14. Orentreich N (1959) Autografts in alopecias and other selected dermatological conditions. Ann Ny Acad Sci 83:463

15. Rassmann WR et al (2002) Follicular Unit Extraction: Minimally Invasive Surgery for Hair Transplantation. Dermatol Surg 28:720-728

16. Unger WP, Shapiro R (2004) Hair Transplantation. Fourth Ed, Revised and Expanded. Marcel Dekker, New York 